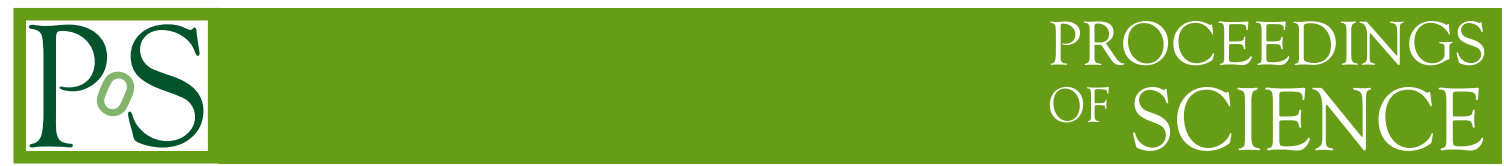

\title{
Search for MSSM Higgs Bosons at the Tevatron
}

\author{
Anton Anastassov* (for the CDF and Dø Collaborations) \\ Rutgers University, USA \\ E-mail: aa@fnal.gov
}

The CDF and DØ Collaborations have performed searches for neutral and charged supersymmetric Higgs bosons produced in $p \bar{p}$ collisions at $\sqrt{s}=1.96 \mathrm{TeV}$. No significant excess of events above the Standard Model backgrounds is observed. The measurements are used to set upper limits on the Higgs production cross sections. The results are interpreted in the framework of the Minimal Supersymmetric extension of the Standard Model.

International Europhysics Conference on High Energy Physics

July 21st - 27th 2005

Lisboa, Portugal

${ }^{*}$ Speaker. 
The Higgs sector in the Minimal Supersymmetric extension of the Standard Model (MSSM) consists of three neutral $(A, h, H)$ and two charged $\left(H^{ \pm}\right)$bosons. One of the neutral bosons $(A)$ is $C P$-odd, and the other two are $C P$-even (assuming $C P$ invariance). At tree level, the Higgs sector is described by the mass of $A\left(m_{A}\right)$, and $\tan \beta=v_{u} / v_{d}$, where $v_{u}$ and $v_{d}$ are the vacuum expectation values of the Higgs fields that couple to up- and down-type particles. For large $\tan \beta$ one of the $C P$ even bosons is nearly mass-degenerate with $A$ and has similar couplings, while the other is SM-like. This paper presents the results of searches for MSSM Higgs bosons produced in $p \bar{p}$ collisions at $\sqrt{s}=1.96 \mathrm{TeV}$ in data collected with the CDF and DØ detectors in Run 2 of the Fermilab Tevatron.

\section{Searches for neutral MSSM Higgs bosons}

The dominant production mechanisms of neutral MSSM Higgs bosons at $p \bar{p}$ colliders are gluon fusion and $b \bar{b}$ fusion. The leading decays are to a pair of $b$-quarks or tau leptons with branching fractions $B(\phi \rightarrow b \bar{b}) \sim 90 \%$ and $B(\phi \rightarrow \tau \tau) \sim 9 \%(\phi=h / H, A)$.

The DØ Collaboration has performed a search for $\phi \rightarrow b \bar{b}$ produced in association with one or two $b$-quarks using a data sample corresponding to an integrated luminosity $\mathscr{L}_{\text {int }}=260 \mathrm{pb}^{-1}$ [1]. The selected events are required to contain at least three, and no more than five jets with transverse energy $E_{T}^{\text {jet }}>15 \mathrm{GeV}$ originating from the same primary vertex. The two leading jets must have $E_{T}^{\text {jet }}>35,20 \mathrm{GeV}$. The $b$-quarks are identified (tagged) by looking for a displaced secondary vertex associated with a jet. The $b$-tagging efficiency reaches $\sim 55 \%$ at $E_{T}^{j e t}=35 \mathrm{GeV}$. The probability to tag a light quark or a gluon jet is $\sim 1 \%$. At least three of the jets in the event must be btagged. Signal acceptance for Higgs bosons in the mass range $m_{A}=90-150 \mathrm{GeV}$ is $0.3-1 \%$. The dominant backgrounds from multi-jet events are estimated from the data. The contributions from other processes such as $Z(\rightarrow b \bar{b})+\mathrm{jets}, Z b$ and $t \bar{t}$ are estimated using MC simulated events. The search for signal is performed by selecting events in mass windows in the di-jet mass distribution of the two leading jets in the event. Limits on the Higgs production cross section are set using a frequentist method - Figure 1.
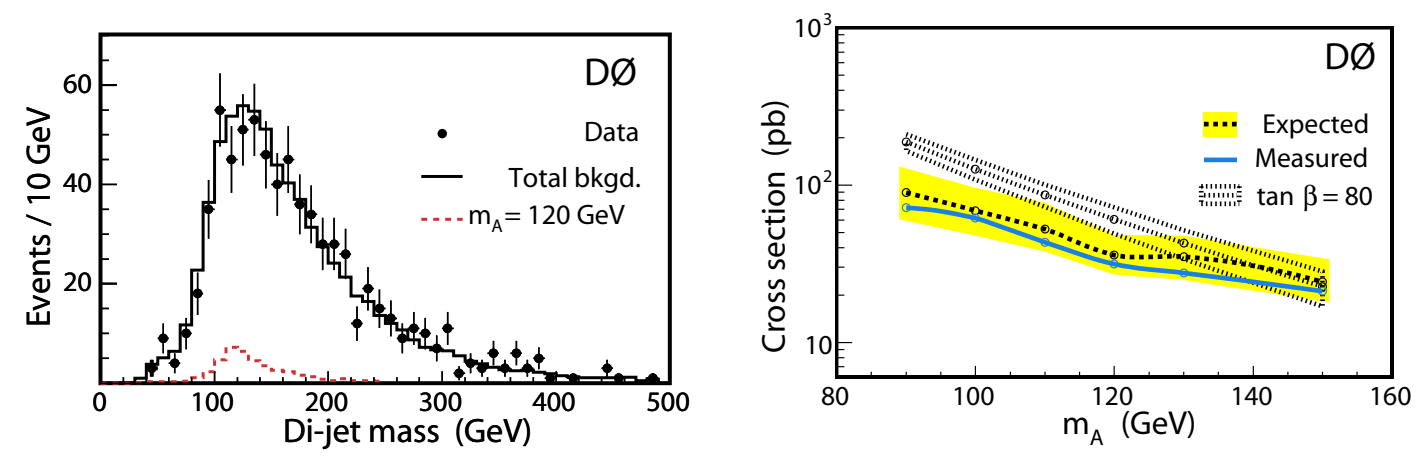

Figure 1: Invariant mass of the two highest- $E_{T}$ jets in the event (left) and upper limit at 95\% CL on neutral MSSM Higgs production cross section as a function of $m_{A}$ (right).

The CDF Collaboration has performed a search for inclusive Higgs production and subsequent decay $\phi \rightarrow \tau \tau$ using a data sample of $\mathscr{L}_{\text {int }}=310 p b^{-1}$ [2]. One $\tau$ is detected in the decay to an $e$ or $\mu$ and neutrinos, and the other in the decay to hadrons and a neutrino. In the following, 
we use $\tau_{e}, \tau_{\mu}$, and $\tau_{\text {had }}$ as shorthand notations for $\tau \rightarrow e v_{e} v_{\tau}, \tau \rightarrow \mu v_{\mu} v_{\tau}$, and $\tau \rightarrow$ hadrons $v_{\tau}$, respectively. The vector sum of the transverse momenta of the neutrinos from $\tau$ decays appears as missing transverse energy $\left(\not_{T}\right)$, determined from the imbalance of energy deposition in the calorimeter. The events in the $\tau_{e} \tau_{\text {had }}\left(\tau_{\mu} \tau_{\text {had }}\right)$ channel are selected by requiring one $e(\mu)$ candidate with transverse momentum $p_{T}^{e(\mu)}>10 \mathrm{GeV}$, and one $\tau_{\text {had }}$ candidate with $p_{T}^{\text {had }}>15 \mathrm{GeV}$ and opposite electric charge. Low-energy multi-jet backgrounds are suppressed by rejecting events with $\left|p_{T}^{e(\mu)}\right|+\left|p_{T}^{h a d}\right|+\left|\not_{T}\right|<50 \mathrm{GeV}$. Backgrounds from $W+$ jet events are suppressed by imposing a requirement on the relative directions of the visible $\tau$ decay products and $H_{T}$. Backgrounds involving quark/gluon jet $\rightarrow \tau_{\text {had }}$ misidentification are estimated from the data. For the remaining sources we use MC simulated events. We expect $496 \pm 5$ (stat) \pm 28 (sys) \pm 25 (lumi) background events (405 from $Z / \gamma^{*} \rightarrow \tau \tau$ ) and observe 487 events in the data. The combined signal acceptance for a Higgs boson of mass $90 \mathrm{GeV}(250 \mathrm{GeV})$ in the $\tau_{e} \tau_{\text {had }}$ and $\tau_{\mu} \tau_{\text {had }}$ channels is $0.8 \%$ (2.0\%). To probe for signal we perform likelihood fits of the partially reconstructed di- $\tau$ mass $m_{v i s}$ formed from the visible $\tau$ decay products and $\not_{T}$. No signal evidence was found and we set 95\% CL exclusion limits on the Higgs production cross section times the branching fraction to $\tau$ pairs - Figure 2 .
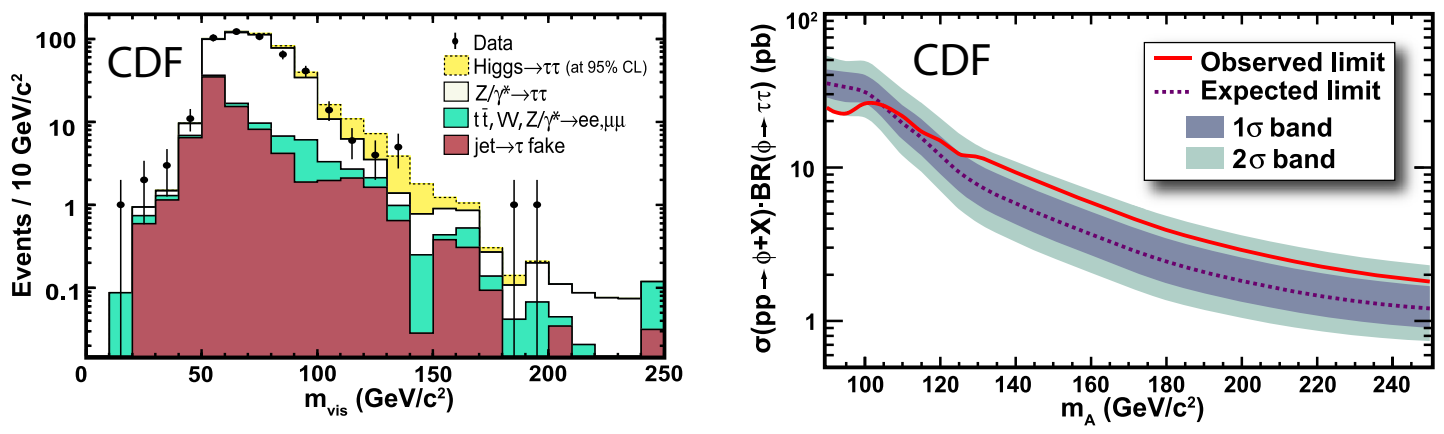

Figure 2: Example fit of $m_{v i s}$ for $m_{A}=140 \mathrm{GeV}$ (left). Upper limit at 95\% CL on neutral MSSM Higgs production cross section times branching fraction to $\tau \tau$ decay as a function of $m_{A}$ (right).

The results of the neutral MSSM Higgs searches are interpreted as exclusions in the $\tan \beta$ vs $m_{A}$ plane for the no-mixing and $m_{h}^{\max }$ benchmark scenarios, with $\mu<0$ and $\mu>0$ [3] - Figure 3.
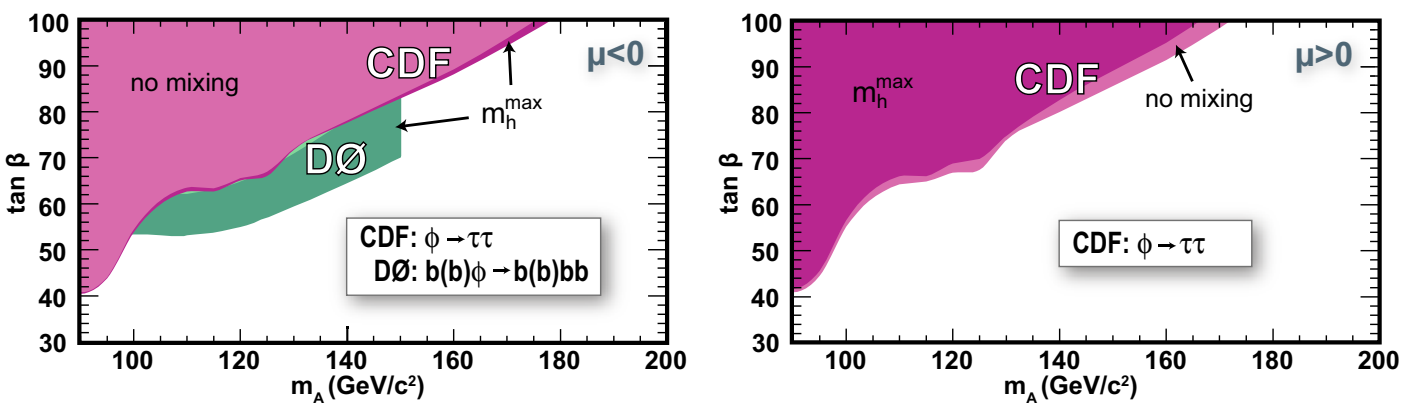

Figure 3: Excluded regions in the $\tan \beta$ vs $m_{A}$ plane from the neutral MSSM Higgs searches at the Tevatron.

\section{Search for $H^{ \pm}$at CDF}

If it is kinematically allowed, $H^{ \pm}$can be produced in top-quark decays and $t \rightarrow b H^{ \pm}$will 
compete with $t \rightarrow b W^{ \pm}$. The decay modes of $H^{ \pm}$and $W^{ \pm}$are different and comparative analysis [4] of the $t \bar{t}$ final states can reveal evidence for $H^{ \pm}$signal. We consider four topological final states: $2 l+$ jets, $l+$ jets $(1 b$-tag), $l+$ jets $(\geq 2 b$-tags), and $l+\tau_{\text {had }}+$ jets $(l=e, \mu)$. Each $t \bar{t}$ candidate event (selected with the criteria in [5]) is uniquely associated with one of these categories. A summary of the estimated backgrounds, expected SM $t \bar{t}$ events, and observed events in a data sample of $\mathscr{L}_{\text {int }}=193 \mathrm{pb}^{-1}$ is shown in Table 1 . In all categories the results are in agreement with the SM. We consider $H^{ \pm}$decays to $\tau v, c s, t^{*} b$ or $W h$, and use a likelihood technique to set limits on parameters of interest. Figure 4 shows two examples of parameter exclusions: $95 \%$ CL limits on $\tan \beta$ for the $m_{h}^{\max }$ benchmark scenario, and quasi model-independent limits on $B\left(t \rightarrow H^{ \pm} b\right)$ when no assumption on the $H^{ \pm}$branching fractions is made.
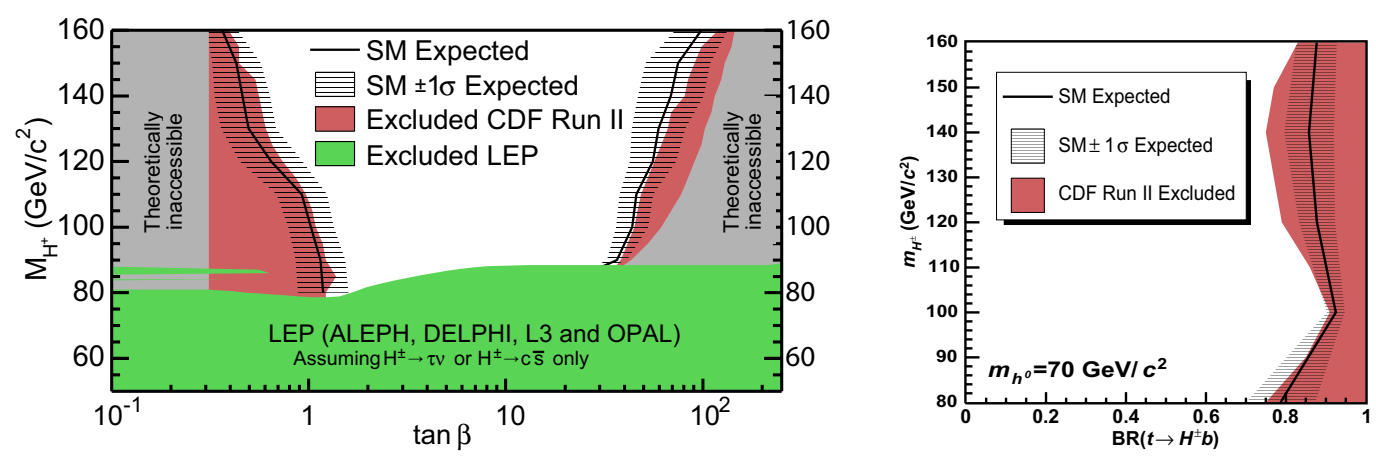

Figure 4: Excluded parameter space for the $m_{h}^{\max }$ scenario (left). Limit on $B\left(t \rightarrow H^{ \pm} b\right)$ at $95 \% \mathrm{CL}$ when no assumption on the $H^{ \pm}$branching fractions is made (right).

\section{Conclusions}

The searches at the Tevatron with data up to $\mathscr{L}_{\text {int }} \sim 300 \mathrm{pb}^{-1}$ have found no evidence for MSSM Higgs bosons. The signal exclusion limits are used to set constraints on MSSM parameters.

\section{References}

[1] V. Abazov et al. (DØ Collaboration), Phys.Rev.Lett. 95, 151801 (2005).

[2] A. Abulencia et al. (CDF Collaboration), hep-ex/0508051 (to be published in PRL) (2005)

[3] M. Carena, S. Heinemeyer, C.E.M. Wagner, and G. Weiglein, hep-ph/9912223 (1999); Eur. Phys. J. C 26, 601 (2003).

[4] A. Abulencia et al. (CDF Collaboration), hep-ex/0510065 (submitted to PRL) (2005).

[5] D. Acosta et al. (CDF Collaboration), Phys. Rev. Lett. 93142001 (2004); Phys. Rev. D 71, 052003 (2005); A. Abulencia et al. (CDF Collaboration), hep-ex/0510063 (2005); 